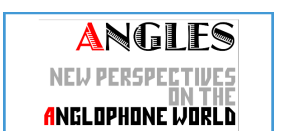

ANELOPHONE WORLI

\section{Angles}

New Perspectives on the Anglophone World

$9 \mid 2019$

Reinventing the Sea

\title{
Graphic Interlude: Reinventing the Sea
}

Geetha Ganapathy-Doré, Ludmila Volná, Walter Raleigh, Aristide Maillol, Joni Sternbach and David Cox

\section{(2) OpenEdition}

\section{Journals}

Electronic version

URL: https://journals.openedition.org/angles/1147

DOI: 10.4000/angles. 1147

ISSN: 2274-2042

\section{Publisher}

Société des Anglicistes de l'Enseignement Supérieur

\section{Electronic reference}

Geetha Ganapathy-Doré, Ludmila Volná, Walter Raleigh, Aristide Maillol, Joni Sternbach and David Cox "Graphic Interlude: Reinventing the Sea", Angles [Online], 9 | 2019, Online since 01 November 2019, connection on 07 June 2022. URL: http://journals.openedition.org/angles/1147 ; DOI: https://doi.org/ 10.4000/angles. 1147

This text was automatically generated on 7 June 2022.

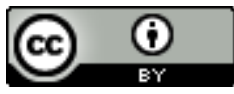

Angles est mise à disposition selon les termes de la Licence Creative Commons Attribution 4.0 International. 


\section{Graphic Interlude: Reinventing the Sea}

Geetha Ganapathy-Doré, Ludmila Volná, Walter Raleigh, Aristide Maillol, Joni Sternbach and David Cox

\section{AUTHOR'S NOTE}

With Anonymous artist

Figure 1. Sir Walter Raleigh's Map of the Indian Ocean in The History of the World, London

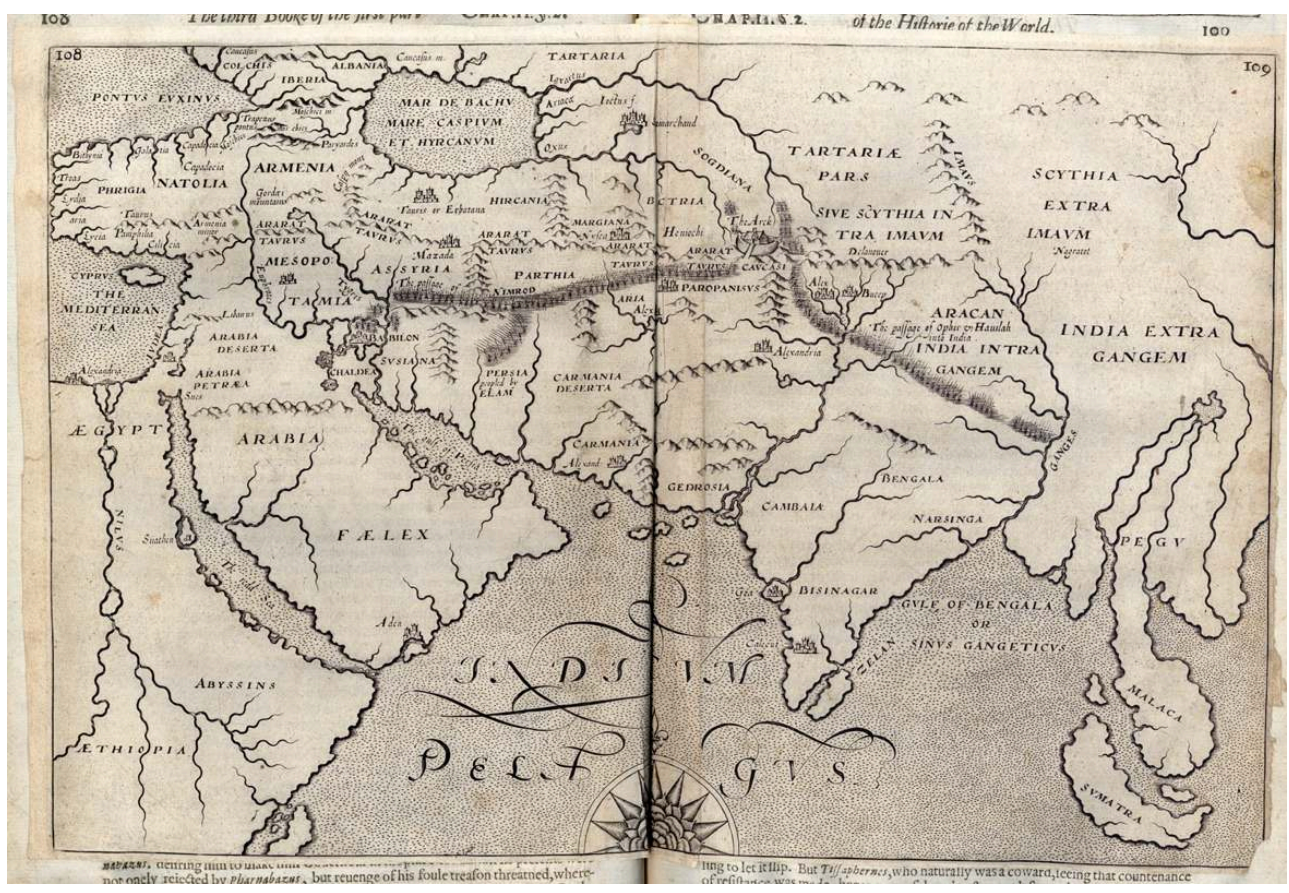

Printed for Walter Burre, 1614, p. 128-9.

Source: https://archive.org/details/historyofworld00rale/page/n12/mode/2up 
Figure 2. Marina Beach Peasant Statues

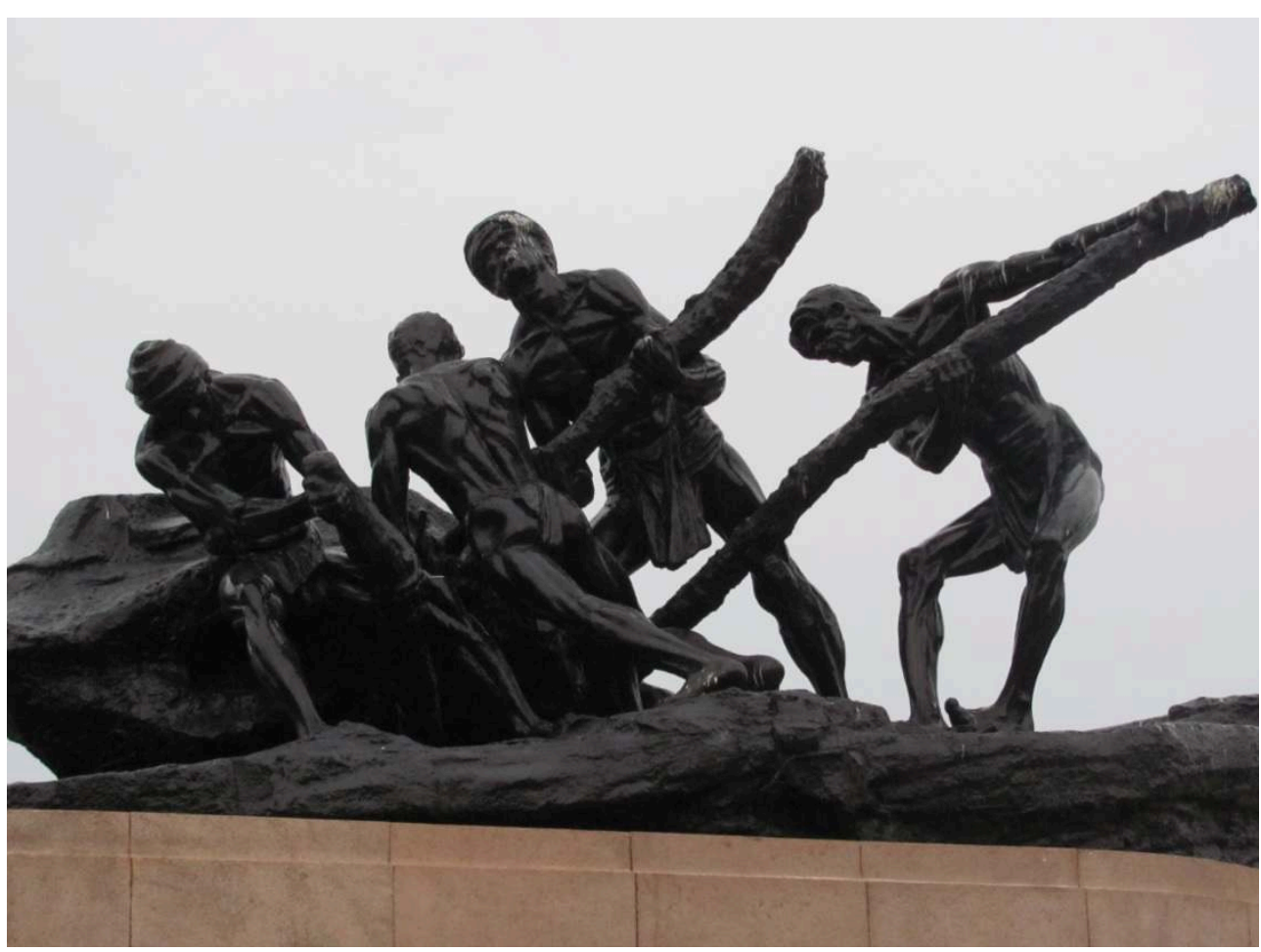

Credit: Geetha Ganapathy-Doré

Figure 3. Muddy footsteps

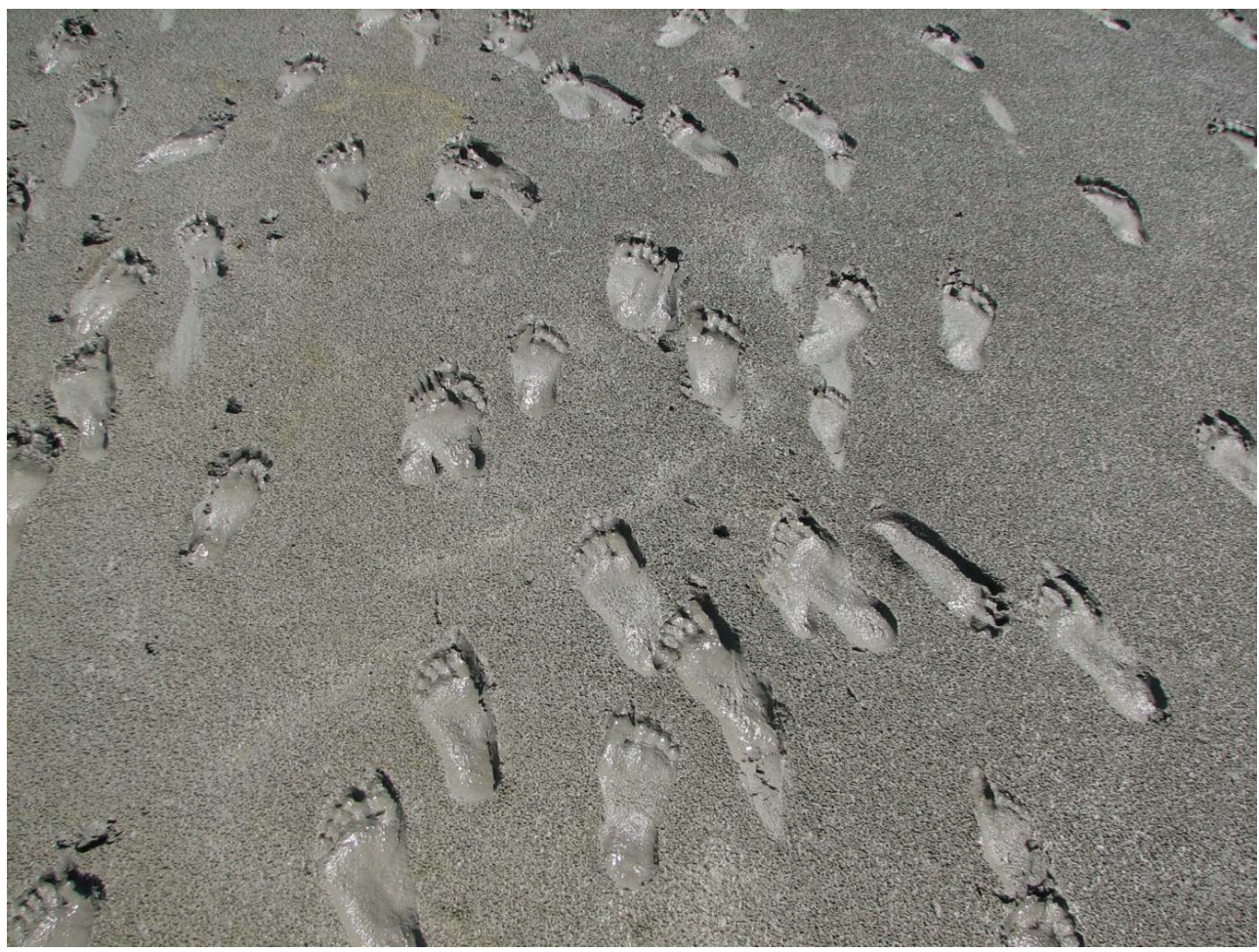

Credit: Ludmila Volná 
Figure 4. The churning of the milk ocean (Rsagara manthana)

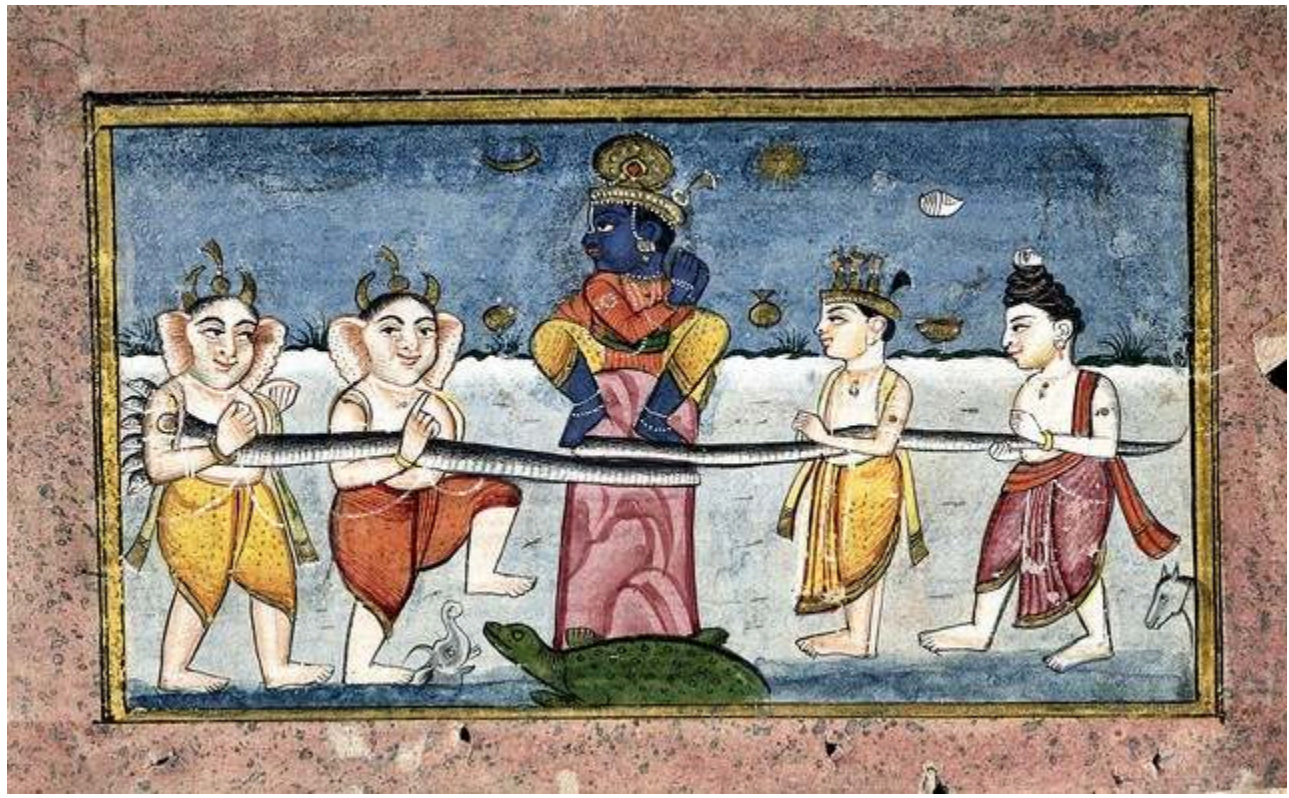

Source: https://wellcomecollection.org/works/s3wypcze

Figure 5. Aristide Maillol, La vague, c. 1898

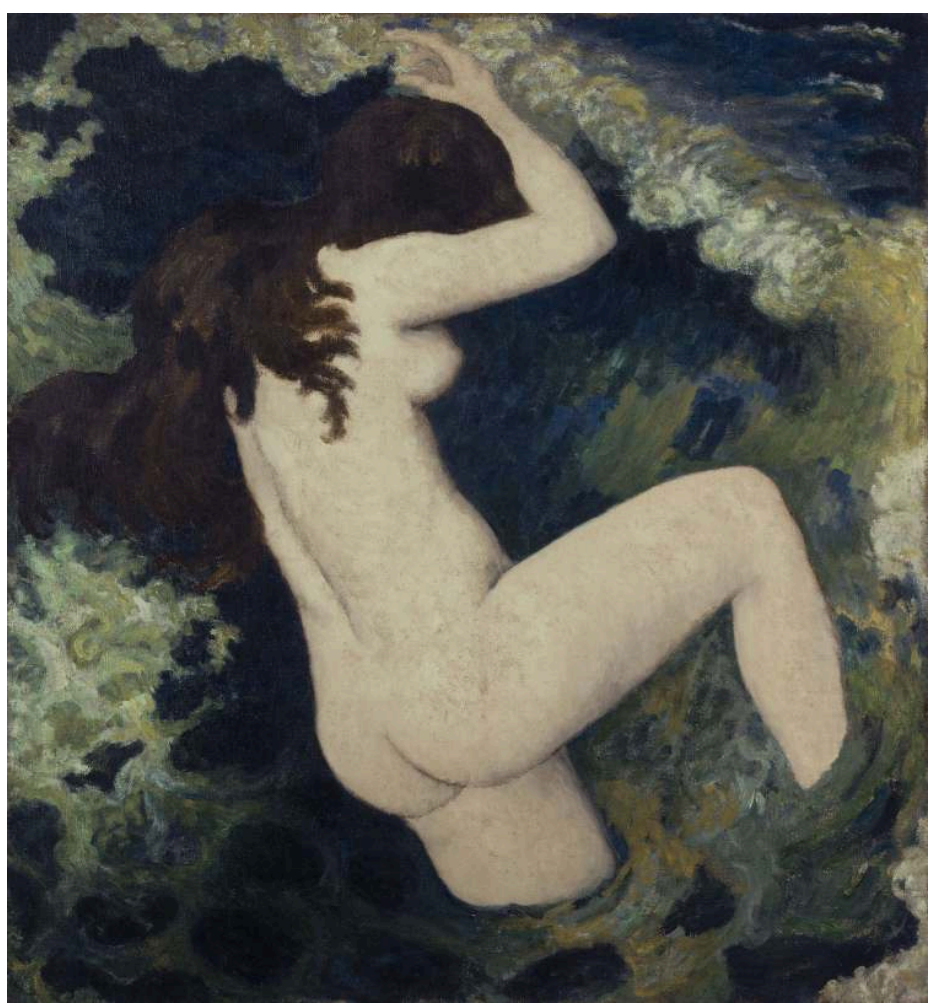

Petit Palais, musée des Beaux-arts de la Ville de Paris. Source: http://parismuseescollections.paris.fr/ $\mathrm{fr} /$ petit-palais/oeuvres/la-vague 
Figure 6. Joni Sternbach. 99.09.18 \#11, from the series "Ocean Details", 1999

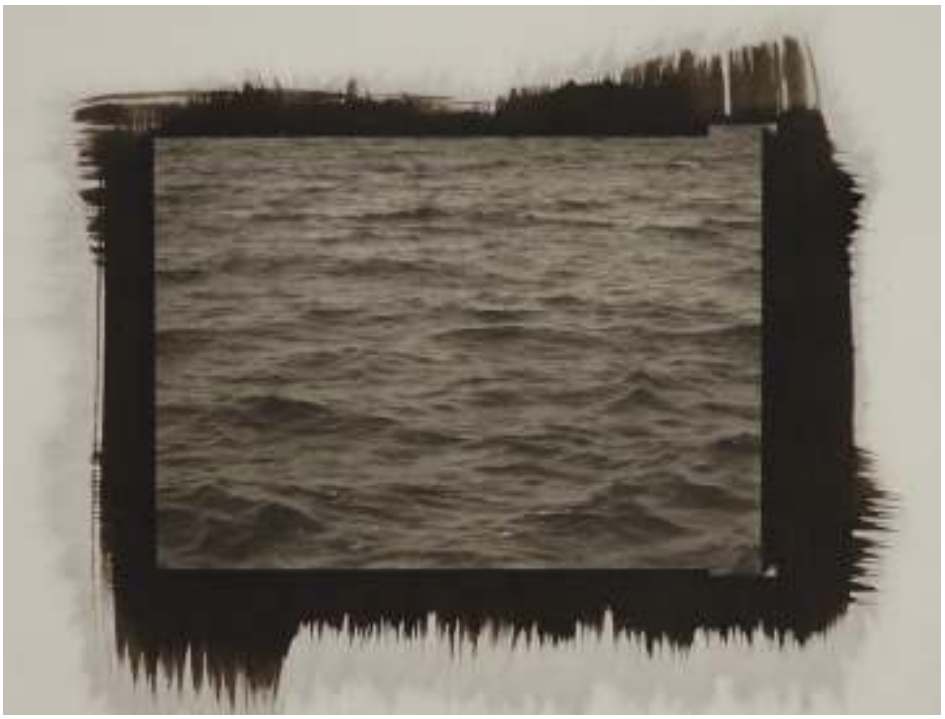

Source: https://www.slam.org/collection/objects/44065

Figure 7. Cover of The Ocean Ferry, January 1934

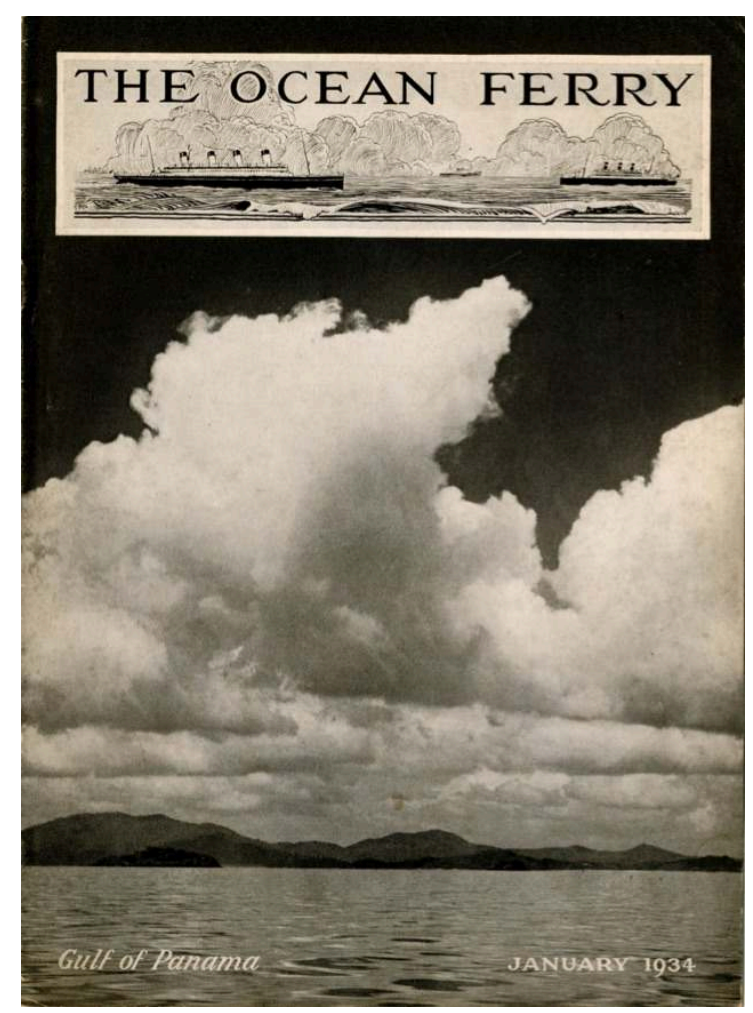

Source: https://dams.antwerpen.be/asset/A1xalXNIMZKbCnTupbZ1dkzy/ I2TaCCHuUhq5gmqGSWzDSoOv 
Figure 8. Two Dragons among Clouds and Waves, Ming Dynasty, 1368-1644

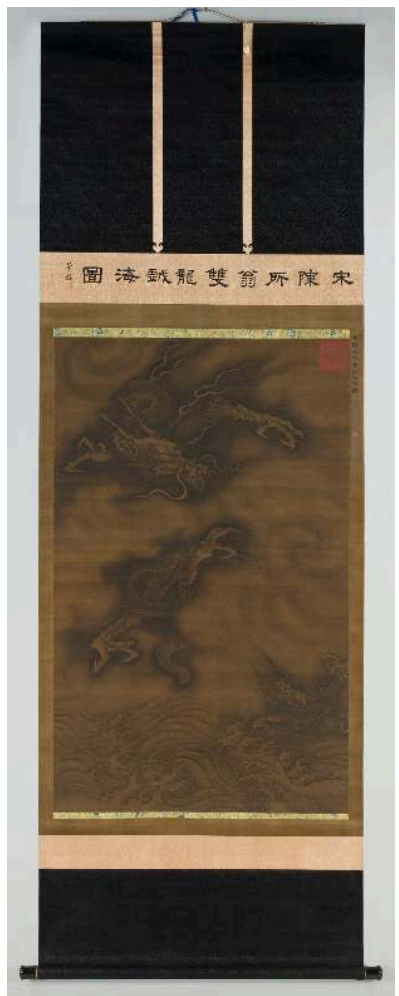

Credit: https://www.slam.org/collection/objects/32680

Figure 9. Cuban Boat, c. 1895-1920

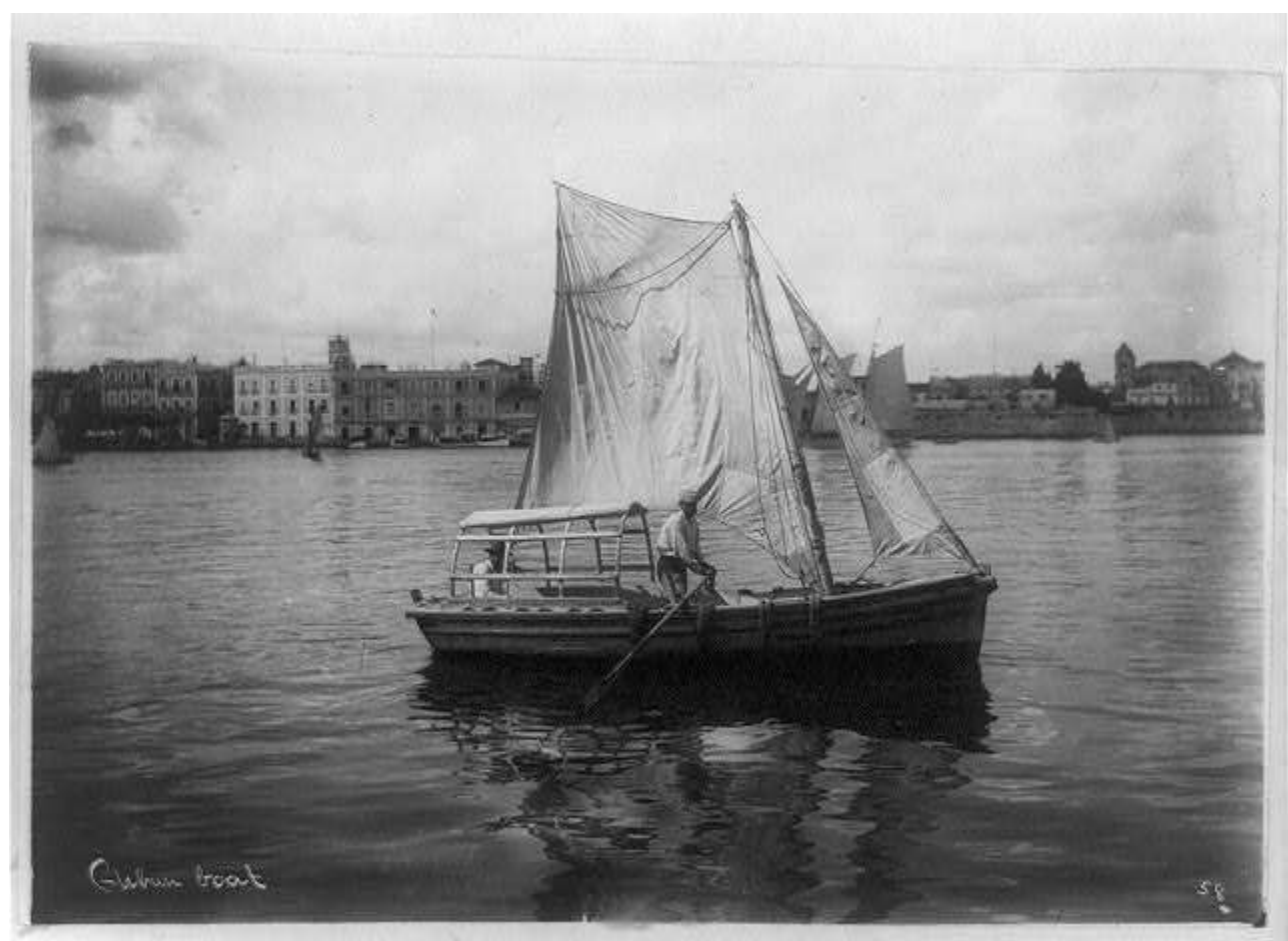

Credit: https://www.loc.gov/item/89708755 
Figure 10. David Cox (1783-1859). Brig and fishing boats at sea; choppy sea with boats sailing right

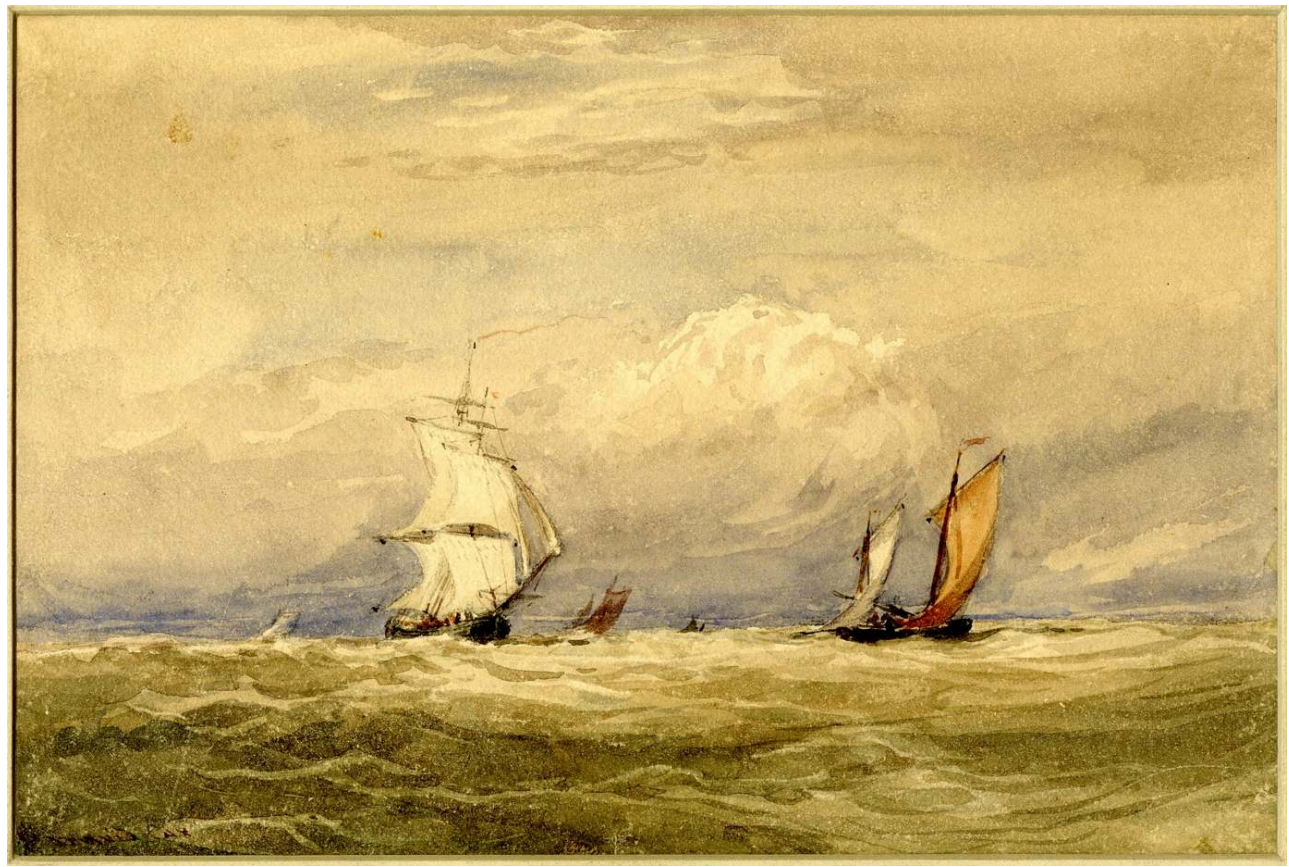

s.d. Courtesy of the British Museum (item 1878,1228.57)

\section{ABSTRACTS}

This graphic interlude features pictures illustrating this issue's topic: the sea as symbol, metaphor and unit of analysis.

Cet interlude graphique est composé d'images qui illustrent le thème de ce numéro: la mer comme symbole, métaphore et unité d'analyse.

\section{INDEX}

Mots-clés: mer, océan, Océan Indien, mythologie, cartographie, bateaux

Keywords: sea, ocean, Indian Ocean, mythology, cartography, boats 